\title{
Value of standard echocardiography at the bedside for COVID-19 patients in intensive care units: a Japanese single-center analysis
}

\author{
Tomoo Nagai $^{1}\left[\right.$ [ Hitomi Horinouchi ${ }^{1} \cdot$ Koichiro Yoshioka $^{1} \cdot$ Yoshihide Nakagawa $^{2} \cdot$ Yuji Ikari $^{1}$
}

Received: 17 May 2021 / Accepted: 9 July 2021 / Published online: 17 August 2021

(c) The Japan Society of Ultrasonics in Medicine 2021

\begin{abstract}
Purpose In the era of COVID-19, those special settings or indications for which standard transthoracic echocardiography (TTE) can safely produce benefits or advantages over minimized ultrasound imaging procedures need to be identified. Thus, the purpose of this study was to conduct a retrospective analysis with offline comprehensive conventional measurement of bilateral heart function and develop an appropriate prognostic model for in-hospital death.

Methods We performed a retrospective analysis of 37 consecutive patients with COVID-19, confirmed by real-time reversetranscriptase polymerase chain reaction assay, who had undergone clinically indicated standard two-dimensional echocardiographic studies in intensive care wards. Offline comprehensive measurement was also performed. We further integrated the echocardiographic findings as paired evidence of vital organ involvement (possible respiratory distress assessed using right ventricular functional parameters, possible myocardial injury assessed using increased wall thickness, effusion or asynergy) and circulatory failure (suspected low flow status assessed using stroke volume index, suspected congestion assessed using elevated right or left atrial pressure). We evaluated its value for in-hospital death along with other echocardiographic findings. Results The most common features included a normal-sized left atrium and left ventricle with preserved left ventricular ejection fraction, despite deteriorated left ventricular flow volume. Less frequent findings, such as abnormalities in the right heart and left ventricular abnormalities suggesting myocarditis, were observed. Although the single echocardiographic parameters failed to show predictive values for in-hospital death, integration of the echocardiographic findings suggested predictive value ( $p=0.04$, odds ratio: 12.28$)$.
\end{abstract}

Conclusion Standard TTE at the bedside with offline comprehensive conventional measurement may provide prognostic information that is valuable for the management of patients with COVID-19.

Keywords COVID-19 $\cdot$ Echocardiography $\cdot$ D-dimer

\section{Introduction}

The first official reports of a severe pneumonia cluster due to an unknown pathogen in Wuhan, China, were released on December 31, 2019 [1, 2]. Soon after the introduction of a quarantine policy towards China, the "Diamond Princess" cruise ship cluster incident hit Japan in early February 2020.

Tomoo Nagai

nagait@tokai.ac.jp

1 Division of Cardiology, Department of Internal Medicine, Tokai University School of Medicine, Shimokasuya 143, Isehara, Kanagawa 259-1193, Japan

2 Department of Emergency and Critical Care Medicine, Tokai University School of Medicine, Shimokasuya 143, Isehara, Kanagawa 259-1193, Japan
In March 2020, the World Health Organization declared the coronavirus disease 2019 (COVID-19) pandemic, which was caused by a novel coronavirus previously called severe acute respiratory syndrome coronavirus 2 (SARS-CoV-2). This emerging virus has been a crucial threat to mankind not only because it had killed more than 2.8 million victims as of the end of March 2021, but also because it heavily affected regional health care systems worldwide [3, 4].

COVID-19 is known to have "Janus" faces targeting two major vital organs, i.e., the lungs and the heart. Transthoracic echocardiography (TTE) plays a prominent role in identifying complications of COVID-19 by evaluating both pulmonary circulation $[5,6]$ and cardiac function [7-9]. However, as TTE studies place sonographers in close proximity to the patient's face, which is within the droplet zone (when coughing for more than $20 \mathrm{~min}$ ), cardiac 
sonographers might have the highest exposure to COVID-19 among healthcare providers. Thus, COVID-19 TTE policies and guidelines were introduced to protect healthcare professionals, especially sonographers, and reduce unnecessary consumption of personal protective equipment (PPE) [10, 11]. In accordance with these policies, elective TTE studies were re-scheduled, "rarely appropriate" TTE examinations were abolished, and the use of limited TTE was encouraged. More point-of-care ultrasound (POCUS) or focused cardiac ultrasound (FOCUS) procedures were performed in the clinical setting $[12,13]$. Quite naturally, the total number of standard TTE studies, including both inpatients and outpatients (not limited to unnecessary examinations), dramatically decreased after the first wave of the COVID-19 pandemic [14]. Even after the release of "reintroduction" guidelines [15], the echocardiography laboratory remained under caution of COVID-19, and did not return to the conditions before the COVID-19 pandemic.

There may be more justifications to conduct "standard" TTE studies apart from as an extravagance in the era of COVID-19. In other words, any special clinical settings or indications for which standard TTE can safely produce benefits or advantages over minimized ultrasound imaging procedures need to be investigated and identified. Some authors have already emphasized the utility of comprehensive measurement using bedside TTE [16]. Many others have developed prognostic models using echocardiographic parameters for worse clinical outcomes among hospitalized patients with COVID-19 [17-19]. However, there are only a few brief reports from Japan, probably due to the relatively small Japanese COVID-19 patient population. At our institution, we have been providing standard two-dimensional (2-D) TTE studies with adequate personal protection [9] at the bedside in intensive care wards for patients with COVID19 by deploying an "on-the-spot scanning alone by a single operator, followed by off-the-spot analysis by anyone" policy ever since the first COVID-19 patients were hospitalized in early February 2020. Thus, the purpose of this study was to conduct a retrospective analysis with comprehensive offline measurement of bilateral heart function and to develop an appropriate prognostic model for in-hospital death using a Japanese COVID-19 cohort.

\section{Materials and methods}

We performed a retrospective analysis of consecutive patients with COVID-19, confirmed by real-time reversetranscriptase polymerase chain reaction assay of nasal swabs, pharyngeal or pulmonary samples, who underwent clinically indicated echocardiographic studies in intensive care wards during their hospitalization at the Tokai University School of Medicine Hospital (Isehara, Kanagawa Prefecture, Japan) between February 2020 and February 2021. Exclusion criteria were: (1) patients younger than 18 years of age and (2) patients with poor echogenicity. This medical institute serves as a tertiary care regional medical center and receives COVID-19 patients with respiratory failure requiring oxygen administration (peripheral arterial oxygen saturation $\leq 93 \%$ on room air), under the medical control of local authorities and the regional government.

All patients were hospitalized in specially dedicated intensive care wards for COVID-19 and received medical management in accordance with current Japanese COVID19 clinical guidelines [20]. The following clinical departments have been responsible for the complete medical management of patients with COVID-19: General Internal Medicine and Family Medicine, Pulmonary Medicine, Emergency and Critical Care Medicine, and Cardiology. The primary clinical endpoint of this study was in-hospital all-cause death. The Institutional Review Board for Clinical Research of the Tokai University School of Medicine approved this observational study protocol. The study was conducted in accordance with the principles outlined in the Declaration of Helsinki. Written informed consent was obtained from all the patients at admission.

Baseline clinical characteristics, laboratory tests, radiological examinations, and treatments were reviewed in the digitalized medical records. The initial values of C-reactive protein, lactic dehydrogenase, white blood cell count, Krebs von den Lungen-6 (KL-6), brain natriuretic peptide (BNP), high-sensitivity troponin (hs-Tpn), and D-dimer levels on admission were collected. In most cases, the measurement of D-dimer was repeated every 2-4 days until the initial decline to obtain the peak values of D-dimer (D-dimer [max]). According to international societies, to reduce the risk of contamination and consumption of personal protective equipment (PPE) during the COVID-19 pandemic, standard TTE was indicated only if there were clinical benefits beyond obtaining patient characteristics, laboratory findings, and conventional bedside images including POCUS or FOCUS. Thus, all bedside TTE services were provided by the medical ultrasound imaging laboratory on the referrals of the physicians who were responsible for the patients' medical management, mainly for the purpose of critical decisionmaking to stabilize the patient's hemodynamic status.

All the standard TTEs were performed at the bedside with patients in the left lateral decubitus, supine, or sitting positions, in quarantined intensive care rooms for COVID-19 patients using a dedicated ultrasound machine (Aplio a/Verifia; CANON Medical Systems Corp., Ohtahara-Shi, Japan) by a single operator with full PPE, who was a validated member of the Japan Society of Ultrasonics in Medicine Registered Medical Sonographer (RMS), following current echocardiographic guidelines [10-12, 21-24]. To reduce the exposure time to the pathogen, the operator was required 
to scan basic two-dimensional (2-D) views (apical views; parasternal long- and short-axis views at the base, mid and apical ventricles; and the subxiphoid view) with or without color Doppler, and both pulse-wave and continuous-wave Doppler images of all valves at the bedside with no on-site measurements, minimally. All images were then transferred via institutional wireless network into the video server of the medical ultrasound laboratory (Prime Vita Plus; NIHON KOHDEN Corp., Shinjuku-ku, Japan). Subsequently, a study review and comprehensive conventional measurement were performed in the medical ultrasound laboratory by at least two RMSs, including the operator, and interpreted by staff cardiologists with or without additional analyses. The final approval for the study was provided by a cardiologist validated as a board-certified fellow of the Japan Society of Ultrasonics in Medicine (FJSUM). Conventional parameters included right ventricular fractional area change (RVFAC), stroke volume index (SVI), maximum tricuspid regurgitation pressure gradient (TRPG), and left myocardial performance index (Tei index). Left ventricular end-diastolic volume (LVEDV) and left ventricular ejection fraction (LVEF) were assessed using the Simpson biplane method. LVEDV was further indexed to body surface area (LVEDVI). We revised the previously reported echocardiographic index for major COVID-19 findings [17, 18], and defined "integrated echocardiographic indicator (IEI)" as the paired evidence of vital organ involvement [a composite of possible respiratory distress (max TRPG $>31 \mathrm{mmHg}$, right ventricular dilatation, RVFAC $\leq 35 \%$ ) and possible myocardial injury (increased left ventricular wall thickness, pericardial effusion, left ventricular a-synergy)] and circulatory failure [a composite of suspected low flow status ( $\mathrm{SVI} \leq 35 \mathrm{ml} / \mathrm{m}^{2}$ ) and suspected congestion (elevated right atrial pressure or left atrial pressure)], as shown in Fig. 1. Elevated right atrial pressure $(8-15 \mathrm{mmHg}$ ) or elevated left atrial pressure (left ventricular diastolic dysfunction grade 2 or 3 ) was diagnosed based on the current guidelines [23, 24]. Right ventricular (RV) dilatation was diagnosed when the max RV diameter exceeded the max left ventricular diameter in the apical fourchamber view [25]. If multiple TTE studies had been performed in a single patient, we considered the study with the most abundant echocardiographic findings as representative.

Numerical data are presented as either the mean \pm standard deviation or the median value, and as numbers (\%) for categorical variables. For categorical variables, the chisquare test was used. Averaged values of continuous variables between the two groups were analyzed by unpaired Student's $t$-test. Ratios of the category values between the two groups were analyzed using the chi-square test. The cutoff values were calculated using receiver operating characteristic (ROC) curve analysis. Considering the sample size, we selected the top three parameters that had a smaller $p$ value less than 0.1 as covariate candidates for multivariate

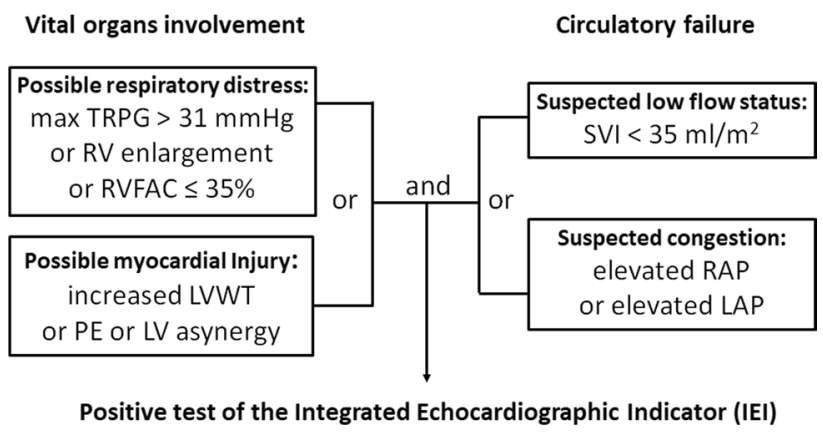

Fig. 1 Algorithm to define the integrated echocardiographic indicator. TRPG tricuspid regurgitation pressure gradient, $R V$ right ventricle, $R V F A C$ right ventricular fractional change, $L V W T$ left ventricular wall thickness, $P E$ pericardial effusion, $L V$ left ventricle, $S V I$ stroke volume index, $R A P$ right atrial pressure, $L A P$ left atrial pressure

analysis, excluding parameters having more than 10 cases with missing values. For multivariate analysis, logistic regression analysis was performed. All statistical analyses were performed using JMP 14.0.0 (SAS Institute, Cary, NC, USA), with $p$ values $<0.05$ considered statistically significant.

\section{Results}

There were 126 admissions with COVID-19 during the study period, of which 37 (29\%) cases had a total of 52 TTE studies referred by each treatment team's physicians. Five TTE studies with poor echogenicity were excluded, as a complete comprehensive echocardiographic measurement could not been performed. Thus, the study group comprised 37 patients with COVID-19 ( 29 men, $70 \pm 12$ years old) and 47 TTE studies. No peri-procedural contamination of bedside TTE was reported. The median time interval from onset to admission was 7.0 days ( 1 st quartile: 5.0 days). The median time interval from onset to general blood sampling at admission was also 7.0 days (1st quartile: 7.0 days). The median time interval from onset to initial sampling for KL-6, BNP, hs-Tpn, and D-dimer was 9.0 days (1st quartile: 6.0 days). The median time interval from onset to peak D-dimer level was 13.0 days ( 1 st quartile: 10.0 days). The median time interval from onset to the representative TTE was 11.0 days (1st quartile: 8.0 days).

Among the 37 COVID-19 cases, 16 (43\%) required intubation for mechanical ventilation at a median 11.0 days from onset. TTE was performed after intubation in 10 patients (27\%). There were 12 cases with cardiovascular complications, including two with cerebral hemorrhage, one with cerebral infarction, one with non-ST-elevated myocardial infarction, and seven with venous thromboembolism. There were 12 cases requiring venous-to-venous extracorporeal 
membranous oxygenation (ECMO) with 11 cases of mortality. Finally, there were 13 cases with an event of all-cause mortality (35\%) at a median of 38.0 days. Direct causes of death included nine cases of multiple organ failure, two cases of cardiogenic shock, one case of sepsis, and one case of respiratory failure.

Among the predisposing factors, laboratory parameters, and electrocardiogram findings, only D-dimer [max] was significantly different between in-hospital death and survival, and no other laboratory measurements, such as hsTpn, KL-6, BNP, and D-dimer levels at admission, were significantly different, as shown in Table 1 . Figure 2 demonstrates the favorable results seen on ROC analysis of D-dimer [max] for predicting in-hospital mortality, having a fair value of area under the curve of 0.88 and $p$ value of $<0.05$, when a cut-off value of $14.2 \mu \mathrm{g} / \mathrm{ml}$ was deployed.
Among the echocardiographic parameters, LVEDV, pulmonary hypertension (max TRPG $>31 \mathrm{mmHg}$, or RV dilatation), RVFAC, elevated right atrial pressure, and IEI were significantly different between in-hospital death and survival (Table 2). There were 20 cases with missing TRPG values, seven cases with missing A wave velocity and $E / A$ ratio values, four cases with missing $e^{\prime}$ and $E / e^{\prime}$ values, and seven cases with missing left atrial pressure values. Figure 3 reveals the most common TTE features of COVID-19, i.e., no left atrium enlargement and left ventricle with preserved LVEF, despite deteriorated LV stroke volume index, and relatively common TTE features, i.e., right heart abnormalities or myocarditis. On multivariate analysis, a model using IEI with the pseudo $R^{2}$ value of 0.35 selected IEI as a possible predictor ( $p=0.04$, odds ratio: 12.28 , confidence interval, 1.19-126.93), as shown in Table 3.
Table 1 Predisposing background and clinical findings

\begin{tabular}{|c|c|c|c|c|}
\hline & All $(n=37)$ & $\begin{array}{l}\text { In-hospital } \\
\text { death } \\
(n=13)\end{array}$ & Survival $(n=24)$ & $p$ value \\
\hline Age, year & $71 \pm 12$ & $73 \pm 12$ & $70 \pm 13$ & 0.44 \\
\hline Male gender, $n(\%)$ & $29(78)$ & $10(77)$ & $19(79)$ & 0.87 \\
\hline Body mass index, $\mathrm{kg} / \mathrm{m}^{2}$ & $24.3 \pm 4.0$ & $25.7 \pm 3.6$ & $23.6 \pm 4.1$ & 0.14 \\
\hline Hypertension, $n(\%)$ & $16(43)$ & $4(31)$ & $12(50)$ & 0.26 \\
\hline Diabetes mellitus, $n(\%)$ & $13(35)$ & $5(38)$ & $8(33)$ & 0.76 \\
\hline Dyslipidemia, $n(\%)$ & $7(19)$ & $3(23)$ & $4(17)$ & 0.64 \\
\hline Coronary artery disease, $n(\%)$ & $5(14)$ & $1(8)$ & $4(17)$ & 0.45 \\
\hline Chronic respiratory disease, $n(\%)$ & $7(19)$ & $3(23)$ & $4(17)$ & 0.64 \\
\hline Smoking habit, $n(\%)$ & $17(46)$ & $7(54)$ & $10(42)$ & 0.48 \\
\hline Hospitalization period, day & $29 \pm 44$ & $48 \pm 69$ & $18 \pm 11$ & $<0.05$ \\
\hline New-onset cardiovascular complications, $n(\%)$ & $12(32)$ & $3(23)$ & $9(38)$ & 0.37 \\
\hline New-onset deep vein thrombosis, $n(\%)$ & $7(19)$ & $2(15)$ & $5(21)$ & 0.69 \\
\hline Use of intravenous corticosteroids & $34(92)$ & $12(92)$ & $21(88)$ & 0.56 \\
\hline Use of antiviral agents & $37(100)$ & $13(100)$ & $24(100)$ & 1.00 \\
\hline Use of anticoagulants & $37(100)$ & $13(100)$ & $24(100)$ & 1.00 \\
\hline Mechanical ventilation, $n(\%)$ & $16(43)$ & $13(100)$ & $3(13)$ & $<0.01$ \\
\hline $\mathrm{ECMO}, n(\%)$ & $12(32)$ & $11(85)$ & $1(4)$ & $<0.01$ \\
\hline Systemic blood pressure at admission, $\mathrm{mmHg}$ & $133 \pm 24$ & $134 \pm 26$ & $132 \pm 23$ & 0.24 \\
\hline D-dimer, $\mu \mathrm{g} / \mathrm{ml}$ & $11.9 \pm 47.8$ & $7.7 \pm 9.7$ & $13.8 \pm 58.4$ & 0.71 \\
\hline D-dimer $[\max ], \mu \mathrm{g} / \mathrm{ml}$ & $37.4 \pm 58.9$ & $63.2 \pm 50.1$ & $23.5 \pm 59.5$ & $<0.05$ \\
\hline $\mathrm{KL}-6, \mathrm{U} / \mathrm{ml}$ & $602 \pm 682$ & $833 \pm 999$ & $468 \pm 347$ & 0.143 \\
\hline $\mathrm{BNP}, \mathrm{pg} / \mathrm{ml}$ & $28 \pm 286$ & $337 \pm 345$ & $235 \pm 239$ & 0.43 \\
\hline High sensitivity troponin $\mathrm{T}, \mathrm{pg} / \mathrm{ml}$ & $95 \pm 198$ & $100 \pm 191$ & $214 \pm 464$ & 0.58 \\
\hline White blood count, $\times 10^{3} / \mu 1$ & $9424 \pm 5165$ & $9414 \pm 5837$ & $9439 \pm 4986$ & 0.91 \\
\hline C-reactive protein, mg/dl & $12.0 \pm 8.9$ & $12.0 \pm 9.7$ & $12.0 \pm 8.7$ & 0.98 \\
\hline Lactate dehydrogenase, $\mathrm{U} / \mathrm{ml}$ & $483 \pm 182$ & $433 \pm 155$ & $510 \pm 192$ & 0.22 \\
\hline Creatinine, mg/dl & $1.82 \pm 3.18$ & $2.52 \pm 3.81$ & $1.43 \pm 2.79$ & 0.33 \\
\hline Heart rate, bpm & $77 \pm 21$ & $83 \pm 27$ & $73 \pm 17$ & 0.20 \\
\hline Rhythm disturbance with atrial fibrillation, $n(\%)$ & $7(19)$ & $2(17)$ & $5(20)$ & 0.43 \\
\hline ST-T abnormality or bundle branch block, $n(\%)$ & $8(22)$ & $5(38)$ & $4(17)$ & 0.18 \\
\hline
\end{tabular}

$E C M O$ extracorporeal membrane oxygenation, $K L-6$ Krebs von den Lungen-6, $B N P$ brain natriuretic peptide 


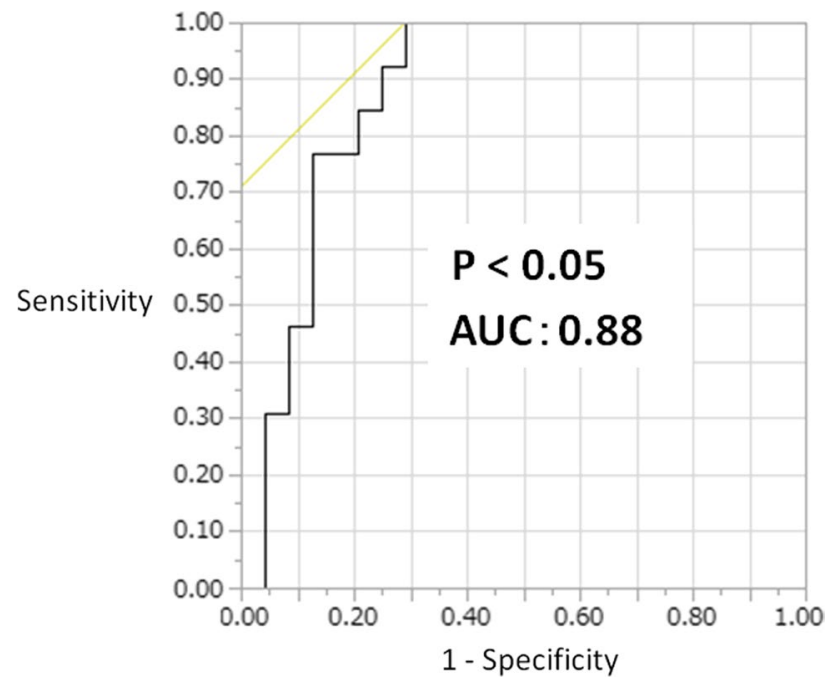

Fig. 2 Receiver operating curve analysis of D-dimer [max] for predicting in-hospital mortality. AUC: area under the curve and obesity with a high body mass index of 32.0. Panel a reveals the typical chest computed tomographic finding of ground-glass opacities in both lower lung lobes. He developed severe respiratory distress 14 days after admission. He received TTE three times, and the final TTE findings are presented. Panel b to panel d reveal typical 2-D TTE findings, such as increased left ventricular wall thickness and small pericardial effusion in front of the right ventricle (panel b and Supplementary video 1), and dilated right ventricle with a flattened interventricular septum indicating right ventricular pressure overload (panel c, panel d, Supplementary videos 2, and 3). Thus, he had a positive IEI result. His initial blood samples on admission revealed the following values: KL-6, $3782 \mathrm{U} / \mathrm{ml}$; D-dimer, $5.7 \mu \mathrm{g} / \mathrm{ml}$; hs-Tpn T, $488 \mathrm{pg} / \mathrm{ml}$; and BNP, 660 pg/ml. His D-dimer [max] reached the initial peak of $71.7 \mu \mathrm{g} / \mathrm{ml}$ 12 days after admission. He received mechanical ventilation as well as ECOM, but he died 42 days after admission.

Figure 4 shows a representative case of a 49 -year-old male with a history of hypertension, diabetes mellitus,

Table 2 Echocardiographic findings

\begin{tabular}{|c|c|c|c|c|}
\hline & All $(n=37)$ & $\begin{array}{l}\text { In-hospital } \\
\text { death }(n=13)\end{array}$ & Survival $(n=24)$ & $p$ value \\
\hline Left atrial volume index, $\mathrm{ml} / \mathrm{m}^{2}$ & $18 \pm 8$ & $16 \pm 7$ & $19 \pm 9$ & 0.27 \\
\hline Left atrial volume index $>34 \mathrm{ml} / \mathrm{m}^{2}$ & $2(5)$ & $0(0)$ & $2(8)$ & 0.28 \\
\hline Interventricular septum, mm & $11 \pm 2$ & $11 \pm 2$ & $11 \pm 2$ & 0.71 \\
\hline Posterior wall thickness, $\mathrm{mm}$ & $10 \pm 2$ & $10 \pm 2$ & $10 \pm 2$ & 0.93 \\
\hline Stroke volume index, $\mathrm{ml} / \mathrm{m}^{2}$ & $32 \pm 9$ & $29 \pm 7$ & $33 \pm 10$ & 0.14 \\
\hline Stroke volume index $\leq 35 \mathrm{ml} / \mathrm{m}^{2}, n(\%)$ & $25(68)$ & $11(85)$ & $14(58)$ & 0.10 \\
\hline LV end-diastolic volume, $\mathrm{ml}$ & $70 \pm 27$ & $55 \pm 21$ & $77 \pm 27$ & 0.01 \\
\hline LV end-diastolic volume index $>74 \mathrm{ml} / \mathrm{m}^{2}$ (male) or $61 \mathrm{ml} / \mathrm{m}^{2}$ (female), $n(\%)$ & $1(3)$ & $1(4)$ & $0(0)$ & 0.46 \\
\hline LV ejection fraction, $\%$ & $69 \pm 10$ & $72 \pm 13$ & $67 \pm 9$ & 0.18 \\
\hline LV ejection fraction $\leq 50 \%, n(\%)$ & $2(5)$ & $1(7)$ & $1(4)$ & 0.65 \\
\hline E wave velocity, $\mathrm{cm} / \mathrm{s}$ & $56 \pm 15$ & $51 \pm 14$ & $59 \pm 15$ & 0.15 \\
\hline A wave velocity, $\mathrm{cm} / \mathrm{s}$ & $70 \pm 21$ & $67 \pm 25$ & $71 \pm 19$ & 0.62 \\
\hline$E / A$ & $0.85 \pm 0.29$ & $0.84 \pm 0.38$ & $0.86 \pm 0.22$ & 0.85 \\
\hline$e^{\prime}, \mathrm{cm} / \mathrm{s}$ & $6 \pm 2$ & $5 \pm 2$ & $6 \pm 1$ & 0.10 \\
\hline$E / e^{\prime}$ & $10.3 \pm 3.1$ & $10.9 \pm 3.4$ & $10.2 \pm 3.0$ & 0.59 \\
\hline Tei index & $0.43 \pm 0.16$ & $0.46 \pm 0.17$ & $0.41 \pm 0.16$ & 0.42 \\
\hline Tei Index $>0.44, n(\%)$ & $16(43)$ & $7(54)$ & $9(38)$ & 0.34 \\
\hline TRPG, mmHg & $28 \pm 10$ & $29 \pm 9$ & $25 \pm 13$ & 0.50 \\
\hline Max TRPG $>31 \mathrm{mmHg}$ or right ventricular dilatation, $n(\%)$ & $8(22)$ & $6(46)$ & $2(8)$ & 0.01 \\
\hline Right ventricular fractional area change, $\%$ & $43 \pm 11$ & $39 \pm 13$ & $45 \pm 10$ & 0.12 \\
\hline Right ventricular fractional area change $\leq 35 \%, n(\%)$ & $8(22)$ & $6(46)$ & $2(8)$ & 0.01 \\
\hline Elevated left atrial pressure, $n(\%)$ & $0(0)$ & $0(0)$ & $0(0)$ & 1.00 \\
\hline Elevated right atrial pressure, $n(\%)$ & $3(8)$ & $3(23)$ & $0(0)$ & 0.01 \\
\hline LV wall thickness $>12 \mathrm{~mm}$ or pericardial effusion or LV a-synergy, $n(\%)$ & $16(43)$ & $7(54)$ & $9(38)$ & 0.34 \\
\hline Positive IEI, $n(\%)$ & $20(54)$ & $12(92)$ & $8(33)$ & $<0.01$ \\
\hline
\end{tabular}

$L V$ left ventricular, TRPG tricuspid regurgitation peak pressure gradient, IEI integrated echocardiographic indicator 


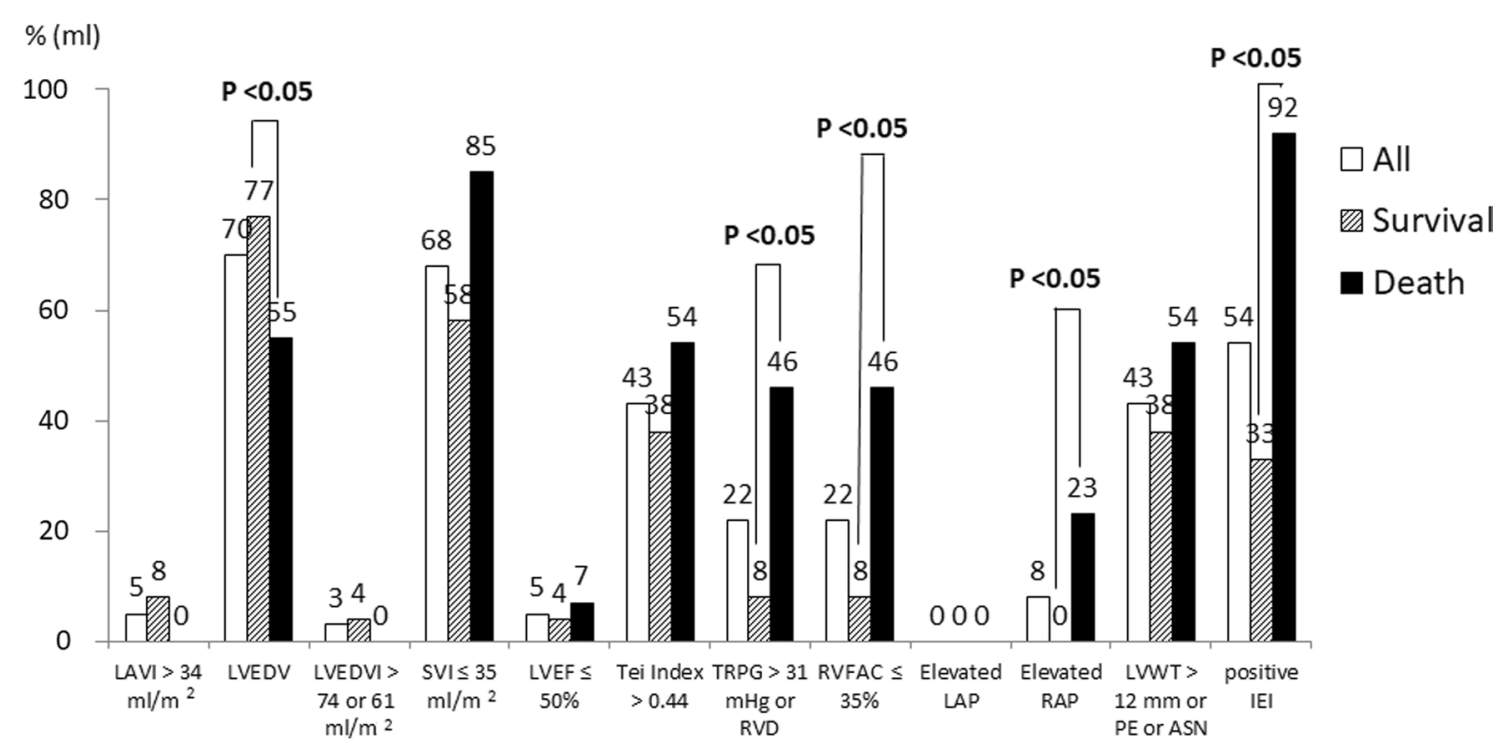

Fig. 3 A bar graph of the typical echocardiographic findings in patients with COVID-19. LAVI left atrial volume index, $L V E D V$ left ventricular end-diastolic volume, $L V E D V I$ left ventricular end-diastolic volume index, $S V I$ stroke volume index, $L V E F$ left ventricular ejection fraction, TRPG tricuspid regurgitation pressure gradient,
$R V D$ right ventricular dilatation, $R V F A C$ right ventricular fractional change, $L A P$ left atrial pressure, $R A P$ right atrial pressure, $L V W T$ left ventricular wall thickness, $P E$ pericardial effusion, $A S N$ a-synergy, $I E I$ integrated echocardiographic indicator

Table 3 Echocardiographic predictors for in-hospital death

\begin{tabular}{|c|c|c|c|c|c|c|}
\hline & \multicolumn{3}{|c|}{ Univariate } & \multicolumn{3}{|c|}{ Multivariate } \\
\hline & $p$ value & Odds ratio & CI $95 \%$ & $p$ value & Odds ratio & CI $95 \%$ \\
\hline D-dimer [max] & $<0.05$ & 1.01 & $1.00-1.03$ & 0.30 & 1.01 & $0.99-1.02$ \\
\hline Left ventricular end-diastolic volume & 0.01 & 0.96 & $0.92-1.00$ & 0.12 & 0.96 & $0.92-1.01$ \\
\hline$e^{\prime}$ & 0.10 & $1.55 \mathrm{e}-21$ & $0-250.71$ & & & \\
\hline Max TRPG $>31 \mathrm{mmHg}$ or RV dilatation & 0.01 & 9.43 & $1.74-75.44$ & NA & NA & NA \\
\hline RV fractional area change $\leq 35 \%$ & 0.01 & 9.43 & $1.74-75.44$ & NA & NA & NA \\
\hline Elevated right atrial pressure & 0.01 & $8.04 \mathrm{e}+8$ & NA & NA & NA & NA \\
\hline Positive IEI & $<0.01$ & 24.00 & $3.75-478.09$ & 0.04 & 12.28 & $1.19-126.93$ \\
\hline
\end{tabular}

$C I$ confidence interval, TRPG tricuspid regurgitation peak pressure gradient, $R V$ right ventricular, $I E I$ integrated echocardiographic indicator, $N A$ not applicable

\section{Discussion}

In this study, we retrospectively investigated the diagnostic value of standard TTE at the bedside, including comprehensive data sets of the cardiac and pulmonary circulation of patients with COVID-19 in intensive care wards, for in-hospital mortality. We reported two main results: (1) Among the standard TTE features of COVID-19 patients at the bedside in intensive care wards, the most common was a normal-sized left atrium and left ventricle with preserved LVEF, despite a deteriorated LV flow volume. There were also other less frequent findings, such as right heart and left ventricular abnormalities, suggestive of myocarditis.
(2) Although all single TTE parameters failed to show predictive value for in-hospital death, integration of TTE findings, i.e., paired lung and heart involvement and circulatory failure, suggested predictive value.

Right heart abnormalities, probably due to respiratory distress, were one of the major TTE findings in critically ill patients with COVID-19 in this study, as well as in previous studies $[5,6,26,27]$. On the other hand, from the early days of the COVID-19 pandemic, numerous studies have reported COVID-19-associated myocardial injury by measuring hs-Tpn [1, 2, 28] and TTE, [7] later confirmed by cardiac magnetic resonance imaging [29], pathology [30], and even in an animal model [31]. Furthermore, a few meta-analyses [32] and some large-scale studies [33-38] to 
Fig. 4 A representative case of a 49-year-old male with a positive integrated echocardiographic indicator result. a Chest computed tomography image, horizontal view. b Echocardiography image, parasternal longaxis view. $\mathbf{c}$ Echocardiography image, parasternal short-axis view. d Echocardiography image, apical four-chamber view

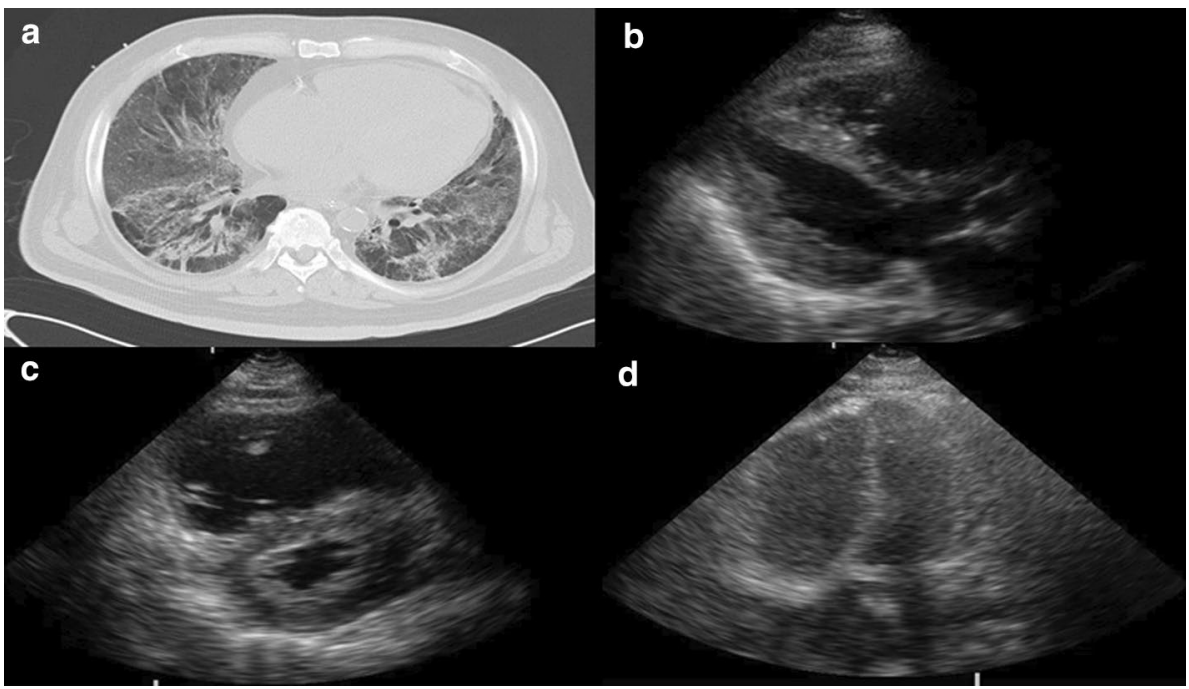

investigate echocardiographic features with conventional parameters with or without strain analysis and their relation to clinical outcomes have already been conducted. In our study, there were five cases (14\%) with a history of coronary artery disease. Additionally, we experienced one non-STelevated myocardial infarction as an apparent acute coronary syndrome. However, we observed 16 patients (43\%) with possible echocardiographic myocardial injury comprised of swollen left ventricular wall, pericardial effusion, and left ventricular a-synergy. For the above reasons, we surmised that COVID-19 myocarditis and focal myocardial infarctions due to COVID-19-induced micro-thrombi were the main causes of myocardial injury. Moreover, the frequent observation of low LV stroke volume index in our study is relatively unique, as only one of the above-mentioned studies included LV stroke volume [36]. It is natural to observe low LV stroke volume in patients with severe pulmonary hypertension or right ventricular dysfunction. However, in our study, there were only 11 cases $(44 \%)$ that demonstrated either pulmonary hypertension or right ventricular dysfunction with right ventricular fractional area change $\leq 35 \%$ among the total of 25 cases with low LV stroke volume index. Considering averagely preserved LV systolic function without valvular pathologies and small left ventricular ventricle, this might be caused by insufficient LV preload, namely dehydration due to COVID-19. Thus, even in patients with preserved LV function, COVID-19 may seriously affect the patient's vital organs via hypo-perfusion caused by dehydration.

In our study, although no single echocardiographic parameter was indicated as a predictor for in-hospital death, we suggest that IEI might have prognostic value for in-hospital death. This is concordant with a previous study by Giustino that concluded that myocardial injury with echocardiographic abnormalities was associated with increased in-hospital mortality [18]. Thus, this might be the main advantage of standard
2-D TTE with comprehensive measurements over a limited ultrasound imaging procedure.

With adequate PPE, no peri-procedural contamination of the referring physicians occurred via bedside TTE while providing comprehensive data sets of the cardiac and pulmonary circulation. Fortunately, our institute was able to maximally prepare for managing COVID-19 patients from the beginning due to the suggestions of local or central government authorities. Thus, since the first TTE case, proper PPE had been used for COVID-19 cases, even before the installation of the COVID-19 guidelines. Additionally, institutional infection control teams have always supervised the education of all healthcare providers, including sonographers, and monitored all infection control practices achieved by healthcare providers.

TTE studies were performed only when ordered by referring physicians responsible for the medical management of patients. Thus, there must be a selection bias in our data, especially in the in-hospital mortality rate. However, it is logical to consider and understand our patients as a subset of COVID-19 patients with cardiac complications and a poor prognosis. We could not conduct offline strain analysis due to the limited capability of the echocardiographic machine. Thus, we are planning to employ other capable machines for this purpose. As the results of this study were based on a single-center database, which included a relatively small number of samples, the conclusions from this study cannot be generalized. However, our findings could provide guidance for larger and/or multicenter prospective studies in the future.

\section{Conclusion}

TTE-derived IEI might have predictive value for in-hospital death in patients with COVID-19 treated in intensive care units. Thus, standard TTE at the bedside with offline 
comprehensive measurements may provide prognostic information that is valuable for the medical management of COVID-19 patients. Further larger, multicenter, prospective studies will be needed in the future.

Supplementary Information The online version contains supplementary material available at https://doi.org/10.1007/s10396-021-01122-1.

Acknowledgements The authors appreciate Professor Yasuhiro Kanatani, Department of Clinical Pharmacology, Tokai University School of Medicine, for his valuable suggestions and statistical interpretations.

\section{Declarations}

\section{Conflict of interest None.}

Ethical statements The Institutional Review Board for Clinical Research of the Tokai University School of Medicine approved the protocol. The study was conducted in accordance with the principles outlined in the Declaration of Helsinki. Written informed consent was obtained from all the patients at admission.

\section{References}

1. Huang C, Wang Y, Li X, et al. Clinical features of patients infected with 2019 novel coronavirus in Wuhan. China Lancet. 2020;395:497-506.

2. Wang D, Hu B, Hu C, et al. Clinical characteristics of 138 hospitalized patients with 2019 novel coronavirus-infected pneumonia in Wuhan, China. JAMA. 2020;323:1061-9.

3. Gralinski LE, Menachery VD. Return of the coronavirus: 2019nCoV. Viruses. 2020;12:135.

4. Zhu N, Zhang D, Wang W, et al. Novel Coronavirus Investigating and Research Team. A novel coronavirus from patients with pneumonia in China, 2019. N Engl J Med. 2020;382:727-33.

5. Fayssoil A, Mustafic H, Mansencal N. The right ventricle in COVID-19 patients. Am J Cardiol. 2020;130:166-7.

6. Argulian E, Sud K, Vogel B, et al. Right Ventricular dilation in hospitalized patients with COVID-19 INFECTION. JACC Cardiovasc Imaging. 2020;13:2459-61.

7. Churchill TW, Bertrand PB, Bernard S, et al. Echocardiographic features of COVID-19 illness and association with cardiac biomarkers. J Am Soc Echocardiogr. 2020;33:1053-4.

8. Capotosto L, Nguyen BL, Ciardi MR, et al. Heart, COVID-19, and echocardiography. Echocardiography. 2020;37:1454-64.

9. Agricola E, Beneduce A, Esposito A, et al. Heart and lung multimodality imaging in COVID-19. JACC Cardiovasc Imaging. 2020;13:1792-808.

10. Kirkpatrick JN, Mitchell C, Taub C, et al. ASE Statement on protection of patients and echocardiography service providers during the 2019 novel coronavirus outbreak: endorsed by the American College of Cardiology. J Am Soc Echocardiogr. 2020;33:648-53.

11. Seo Y, Daimon M, Yamada H, et al. Public Relations Committee, Guidelines Committee of the Japanese Society of Echocardiography. Review of the efforts of the Japanese Society of Echocardiography for coronavirus disease 2019 (COVID-19) during the initial outbreak in Japan. J Echocardiogr. 2020;18:226-33.

12. Johri AM, Galen B, Kirkpatrick JN, Lanspa M, et al. ASE statement on point-of-care ultrasound during the 2019 novel coronavirus pandemic. J Am Soc Echocardiogr. 2020;33:670-3.
13. Schrift D, Barron K, Arya R, et al. The Use of POCUS to Manage ICU Patients with COVID-19. J Ultrasound Med. 2020. https:// doi.org/10.1002/jum.15566 (Online ahead of print).

14. Ward RP, Lee L, Ward TJ, et al. Utilization and appropriateness of transthoracic echocardiography in response to the COVID-19 pandemic. J Am Soc Echocardiogr. 2020;33:690-1.

15. Hung J, Abraham TP, Cohen MS, et al. ASE statement on the reintroduction of echocardiographic services during the COVID19 pandemic. J Am Soc Echocardiogr. 2020;33:1034-9.

16. Bursi F, Santangelo G, Sansalone D, et al. Prognostic utility of quantitative offline 2D-echocardiography in hospitalized patients with COVID-19 disease. Echocardiography. 2020;37:2029-39.

17. Sud K, Vogel B, Bohra C, et al. Echocardiographic findings in patients with COVID-19 with significant myocardial injury. J Am Soc Echocardiogr. 2020;33:1054-5.

18. Giustino G, Croft LB, Stefanini GG, et al. Characterization of myocardial injury in patients with COVID-19. J Am Coll Cardiol. 2020;76:2043-55.

19. Doyen D, Dupland P, Morand L, et al. Characteristics of cardiac injury in critically ill patients with coronavirus disease 2019 . Chest. 2020;S0012-3692:35109-16.

20. Japan the Ministry of Health, Labour and Welfare and the National Institute of Infectious Diseases. Clinical Management of Patients with COVID-19: A Guide for Front-Line Healthcare Workers Version 4.2 (in Japanese). https://www.mhlw.go.jp/content/00074 2297.pdf. Accessed 31 Mar 2021.

21. Mitchell C, Rahko PS, Blauwet LA, et al. Guidelines for performing a comprehensive transthoracic echocardiographic examination in adults: recommendations from the American Society of Echocardiography. J Am Soc Echocardiogr. 2019;32:1-64.

22. Doherty JU, Kort S, Mehran R, et al. ACC/AATS/AHA/ASE/ ASNC/HRS/SCAI/SCCT/SCMR/STS 2019 Appropriate Use Criteria For Multimodality Imaging in the Assessment of Cardiac Structure and Function in Nonvalvular Heart Disease: A Report of the American College of Cardiology Appropriate Use Criteria Task Force, American Association for Thoracic Surgery, American Heart Association, American Society of Echocardiography, American Society of Nuclear Cardiology, Heart Rhythm Society, Society For Cardiovascular Angiography and Interventions, Society of Cardiovascular Computed Tomography, Society for Cardiovascular Magnetic Resonance, and the Society of Thoracic Surgeon. J Am Coll Cardiol. 2019;73:488-516.

23. Lang RM, Badano LP, Mor-Avi V, et al. Recommendations for cardiac chamber quantification by echocardiography in adults: an update from the American Society of Echocardiography and the European Association of Cardiovascular Imaging. J Am Soc Echocardiogr. 2015;28:1-39.e14.

24. Nagueh SF, Smiseth OA, Appleton CP, et al. Recommendations for the evaluation of left ventricular diastolic function by echocardiography: an update from the American Society of Echocardiography and the European Association of Cardiovascular Imaging. J Am Soc Echocardiogr. 2016;29:277-314.

25. Galiè N, Humbert M, Vachiery JL, et al. 2015 ESC/ERS Guidelines for the diagnosis and treatment of pulmonary hypertension: The Joint Task Force for the Diagnosis and Treatment of Pulmonary Hypertension of the European Society of Cardiology (ESC) and the European Respiratory Society (ERS): Endorsed by: Association for European Paediatric and Congenital Cardiology (AEPC), International Society for Heart and Lung Transplantation (ISHLT). Eur Heart J. 2016;37:67-119.

26. Bleakley C, Singh S, Garfield B, et al. Right Ventricular dysfunction in critically ill COVID-19 ARDS. Int J Cardiol. 2021;327:251-8.

27. D'Alto M, Marra AM, Severino S, et al. Right ventricular-arterial uncoupling independently predicts survival in COVID-19 ARDS. Crit Care. 2020;24:670. 
28. Nie SF, Yu M, Xie TY, et al. Cardiac Troponin I is an independent predictor for mortality in hospitalized patients with COVID-19. Circulation. 2020;142:608-10.

29. Luetkens JA, Isaak A, Zimmer S, et al. Diffuse myocardial inflammation in COVID-19 associated myocarditis detected by multiparametric cardiac magnetic resonance imaging. Circ Cardiovasc Imaging. 2020;13:e010897.

30. Pellegrini D, Kawakami R, Guagliumi G, et al. Microthrombi as a major cause of cardiac injury in COVID-19: a pathologic study. Circulation. 2021;143:1031-42.

31. Siddiq MM, Chan AT, Miorin L, et al. Physiology of cardiomyocyte injury in COVID-19. medRxiv. 2020. https://doi.org/10.1101/ 2020.11.10.20229294.

32. Shafiabadi Hassani N, Shojaee A, Khodaprast Z, et al. Echocardiographic features of cardiac injury related to COVID-19 and their prognostic value: a systematic review. J Intensive Care Med. 2021;36:500-8.

33. Dweck MR, Bularga A, Hahn RT, et al. Global evaluation of echocardiography in patients with COVID-19. Eur Heart J Cardiovasc Imaging. 2020;21:949-58.

34. Pagnesi M, Baldetti L, Beneduce A, et al. Pulmonary hypertension and right ventricular involvement in hospitalized patients with COVID-19. Heart. 2020;106:1324-31.
35. Mahmoud-Elsayed HM, Moody WE, Bradlow WM, Khan-Kheil $\mathrm{AM}$, et al. Echocardiographic findings in patients with COVID-19 pneumonia. Can J Cardiol. 2020;36:1203-7.

36. Szekely Y, Lichter Y, Taieb P, et al. Spectrum of cardiac manifestations in COVID-19: a systematic echocardiographic study. Circulation. 2020;142:342-53.

37. Li Y, Li H, Zhu S, et al. Prognostic value of right ventricular longitudinal strain in patients with COVID-19. JACC Cardiovasc Imaging. 2020;13:2287-99.

38. Lassen MCH, Skaarup KG, Lind JN, et al. Echocardiographic abnormalities and predictors of mortality in hospitalized COVID-19 patients: the ECHOVID-19 study. ESC Heart Fail. 2020;7:4189-97.

Publisher's Note Springer Nature remains neutral with regard to jurisdictional claims in published maps and institutional affiliations. 